\title{
Flora vascular de la Laguna Avendaño, Provincia de Diguillín, Chile
}

\section{Vascular flora of the Avendaño Lagoon, Province of Diguillín, Chile}

\author{
Carlos Baeza ${ }^{1 *}$, Roberto Rodriguez ${ }^{1} \&$ Oscar Toro-Núñez ${ }^{1}$ \\ 1'Departamento de Botánica, Facultad de Ciencias Naturales y Oceanográficas, Universidad de Concepción, Concepción, \\ Chile. \\ *cbaeza@udec.cl
}

\begin{abstract}
RESUMEN
La Laguna Avendaño se ubica en la Provincia de Diguillín, dentro del macrobioclima Mediterráneo, Región de Ñuble, Chile, y constituye un importante centro de recreación durante los meses de verano. Se estudió la flora vascular presente en el cuerpo de agua y en sectores aledaños, los cuales difieren en el grado de antropización. Se compararon 5 sitios en cuanto a la composición y riqueza específica de ellos. Los sitios más alterados, en base al número de especies introducidas, corresponden a los lugares abiertos al público y de uso recreacional masivo. Se documenta la presencia de 113 especies de plantas vasculares que crecen espontáneamente, incluyendo 6 Pteridophyta, 77 Dicotyledoneae y 30 Monocotyledoneae. Del total de especies, 13,3\% son endémicas de Chile, 52,2\% nativas y 34,5\% introducidas. Las familias mejor representadas son: Poaceae, Asteraceae, Cyperaceae y Scrophulariaceae. El objetivo de este catálogo fue describir la flora aledaña al cuerpo de agua de esta laguna que tiene una enorme importancia turística para la Comuna de Quillón, y por ende fuerte presión antrópica.
\end{abstract}

Palabras clave: Laguna Avendaño, flora vascular, Chile.

\begin{abstract}
The Avendaño Lagoon is located in the Province of Diguillin, Nuble Region, Chile and represents a very popular recreation area during the summer season. This note reports a floral list of the Lagoon Avendaño and its environs, which differ in their levels of anthropization. We compared five sites by measuring composition and specific richness of the vegetation associated to them. The most altered sites, based on the number of introduced species, corresponded to open and recreational areas. Herein, 113 spontaneous species of vascular plants are documented, including 6 Pteridophyta, 77 Dicotyledoneae, and 30 Monocotyledoneae. From these, 13.3\% are endemic, 52.2 native, and 34.5\% alien species. The most frequently represented families were Poaceae, Asteraceae, Cyperaceae and Scrophulariaceae. The purpose of this catalog was to describe the flora of vascular plant surrounding the body of water of this lagoon which has a large tourist importance for the Commune of Quillón, linking the plant composition with the land uses in its borders.
\end{abstract}

KEYwords: Lake Avendaño, vascular flora, Chile.

\section{INTRODUCCIÓN}

La Laguna Avendaño es un cuerpo de agua dulce que está en la comuna de Quillón, Región de Nuble, en la zona correspondiente al límite sur del macrobioclima Mediterráneo, es decir, templado-cálido con estación seca y lluviosa de igual duración (Luebert \& Pliscoff 2006), y desde el punto de vista vegetacional está incluido dentro de la Región del Bosque Caducifolio Maulino (Gajardo 1994). La Laguna Avendaño está circundada en la actualidad por suelos agrícolas, terrenos destinados a la ganadería, viñedos, pastizales de temporada y cultivos de diversos tipos a baja escala. La laguna es una importante alternativa de recreación, por su proximidad geográfica, para la población de las provincias de Concepción, Diguillín y Biobío. En los meses de verano el pueblo de Quillón (17.485 habitantes) triplica su población por el flujo de turistas. El creciente aumento del número de casas residenciales y de balnearios en el entorno de la laguna ha aumentado la eutroficación del agua (Dellarossa 1998). En la actualidad existen sectores no aptos para el baño ni para la recreación por el intenso crecimiento que presentan los cinturones litorales de vegetación acuática. Existe muy poca información publicada acerca de la vegetación en las riberas de las numerosas lagunas presentes en Chile y específicamente en la zona centro sur estas lagunas experimentan una creciente presión por uso recreacional. Solamente se pueden destacar los trabajos sobre flora y vegetación de la laguna el Peral, 
Región de Valparaíso, Chile (Ramírez et al. 1987), de la vegetación del río Purapel, en la Región del Maule (San Martín 1989), sobre la vegetación de las marismas del centro sur de Chile (San Martín et al. 1992) y sobre la flora y vegetación del estero de Reñaca, Región de Valparaíso (San Martín et al. 2001); todas en la parte central o norte de la zona Mediterránea del país. En este contexto, con el objetivo de conocer la diversidad vegetal presente en un cuerpo de agua que ha sufrido mucha presión antrópica en las últimas décadas, se hizo un estudio de la flora vascular presente en las riberas de la laguna Avendaño (Región de Nuble). Esto, considerando las plantas arraigadas al sustrato, las libres flotantes y la flora circundante al cuerpo de agua ubicada hasta el nivel más alto de inundación que alcanza el espejo de agua en los meses de invierno. Se discute la relación entre la composición de la flora y el tipo de actividad antrópica que se desarrolla en la ribera.

\section{MATERIALES Y MÉTODOS}

La Laguna Avendaño ( $36^{\circ} 45^{\prime} \mathrm{S}-72^{\circ} 27^{\prime} \mathrm{O}, 66$ m s.n.m.) tiene una superficie de $1.753 .172 \mathrm{~m}^{2}$. Se ubica en una meseta disectada, con una altura media no superior a los $500 \mathrm{~m}$ (Fuenzalida 1965). El área de estudio de este trabajo incluye cinco zonas de muestreo ubicadas en diferentes sectores de la laguna (Fig. 1). Estos sitios son representativos del estado actual del perímetro de la laguna y representan distintos niveles de antropización: Balneario Municipal (A); frente al Balneario Municipal, Patio Los Naranjos (B); Propiedad del Dr. Ferrari (C); entrada Brazo Bravo (E) y sector Antupahuil (D). En cada zona de muestreo se hizo un relevamiento de toda la vegetación presente, posteriormente el material recolectado se depositó en el herbario del Departamento de Botánica de la Universidad de Concepción (CONC). Se realizaron numerosas expediciones a los puntos de muestreo durante 18 meses (entre 2002 - 2003) para cubrir todas las estaciones del año. Para la confección del catálogo, basado en la clasificación de Cronquist (1981), se siguió el modelo de Baeza et al. (1999) y Rodríguez et al. (2008): determinación de cada ejemplar utilizando claves dicotómicas y comparando el material recolectado con las muestras depositadas en el Herbario CONC. Estimamos la abundancia relativa porcentual de especies de cada sitio en estudio, para comparar la diversidad taxonómica entre sitios y la similitud en la composición de estos utilizando el índice de Sørensen-Dice (Dice 1945, Sørensen 1948). Las distancias entre sitios fueron organizadas en un dendrograma con el criterio de agrupamiento a través de una media aritmética no ponderada (UPGMA; Sneath \& Sokal
1975), controlando el nivel de distorsión con el índice de correlación cofenética (Sokal \& Rolph 1962). El cálculo de matrices de similitud y dendrogramas fueron realizados con PAST v. 3.15 (Hammer et al. 2001). Se realizó un análisis de perfil de similitud (SIMPROF) para determinar cuáles de los sitios muestreados eran significativamente diferentes dada su composición taxonómica (Clarke et al. 2008). Este análisis se realizó con 999 permutaciones y un umbral de significancia del 5\%. Además fue realizado con los paquetes ade4 (Dray \& Dufour 2007), clustsig (Whitaker \& Christman 2014) y vegan (Oksanen et al. 2017) en la plataforma estadística R ver. 3.4 (R Core Team 2017).

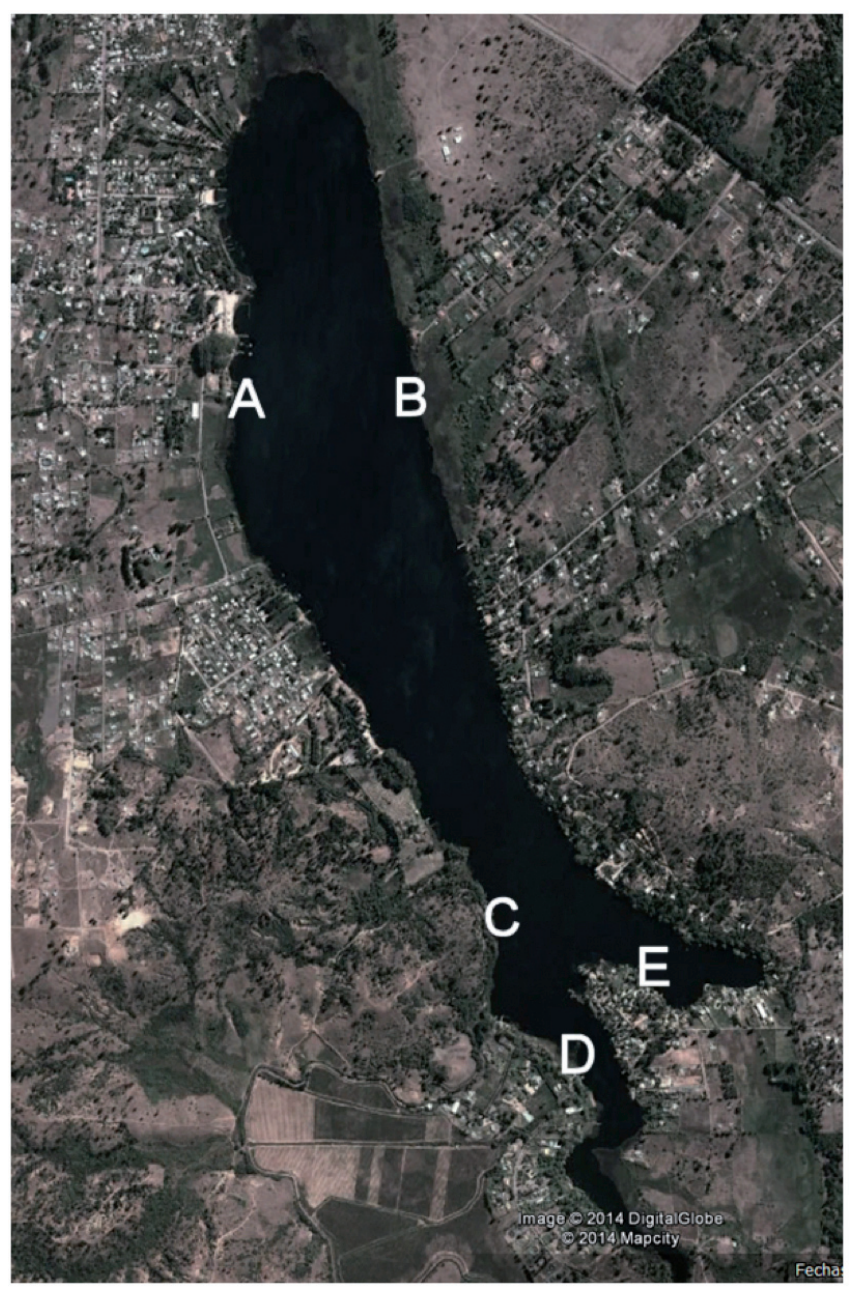

Figura 1. Mapa Laguna Avendaño y áreas de muestreo (A-E). / Map of the Lagoon Avendaño and sampling areas (A-E). 


\section{RESULTADOS}

RiQUEZA FLORÍSTICA

La flora de plantas vasculares de la Laguna Avendaño y su entorno cercano alcanza a 113 especies. La distribución en categorías taxonómicas superiores se muestra en la Tabla 1; el grupo con mayor cantidad de especies corresponde a las Dicotyledoneae (77 especies, 68.14\%), que concentra también la mayor cantidad de especies introducidas (34 especies). Dentro de las Monocotyledoneae, la familia Poaceae presenta cinco especies introducidas, lo que indica la fuerte influencia antrópica en la transformación del paisaje natural.

Las familias de plantas vasculares con un número mayor o igual a cinco especies se muestran en la Tabla 2. Las familias con mayor número de especies son Asteraceae (12 especies) y Poaceae (12 especies), la familia Cyperaceae es la que posee mayor cantidad de especies nativas (7 especies). Estas plantas tienen su hábitat en suelos muy húmedos, inundados en invierno. La familia Myrtaceae está representada aquí por 4 especies que son árboles y arbustos netamente hidrófilos.
FORMAS DE VIDA

En la laguna Avendaño se encuentran representadas cinco formas de vida, según la clasificación de Raunkaier (1934). El grupo más diversificado son los Hemicriptófitos con 35 (31\%) especies (Fig. 2), la que agrupa a plantas con hojas basales formando una roseta o forman matas densas y compactas habitando de preferencia a la sombra de los árboles y arbustos. Siguen en importancia los Fanerófitos (27 especies, 23,9\%), donde destacan los géneros Luma y Myrceugenia que tienen representantes arbóreos asociados a ambientes bastante húmedos; los Geófitos están representados por $16(14,2 \%)$ especies que en gran medida son plantas palustres cuyas yemas de renuevo pasan parte del año enterradas en el barro. Las plantas anuales o Terófitos son relativamente escasas (21 especies, 18,6\%) y el número puede variar con los cambios de uso del suelo al estar expuesto al tránsito de las personas que visitan el lugar; por último, los Hidrófitos que agrupan a las plantas acuáticas cuyas yemas de renuevo se encuentran bajo el agua (14 especies, 12,4\%).

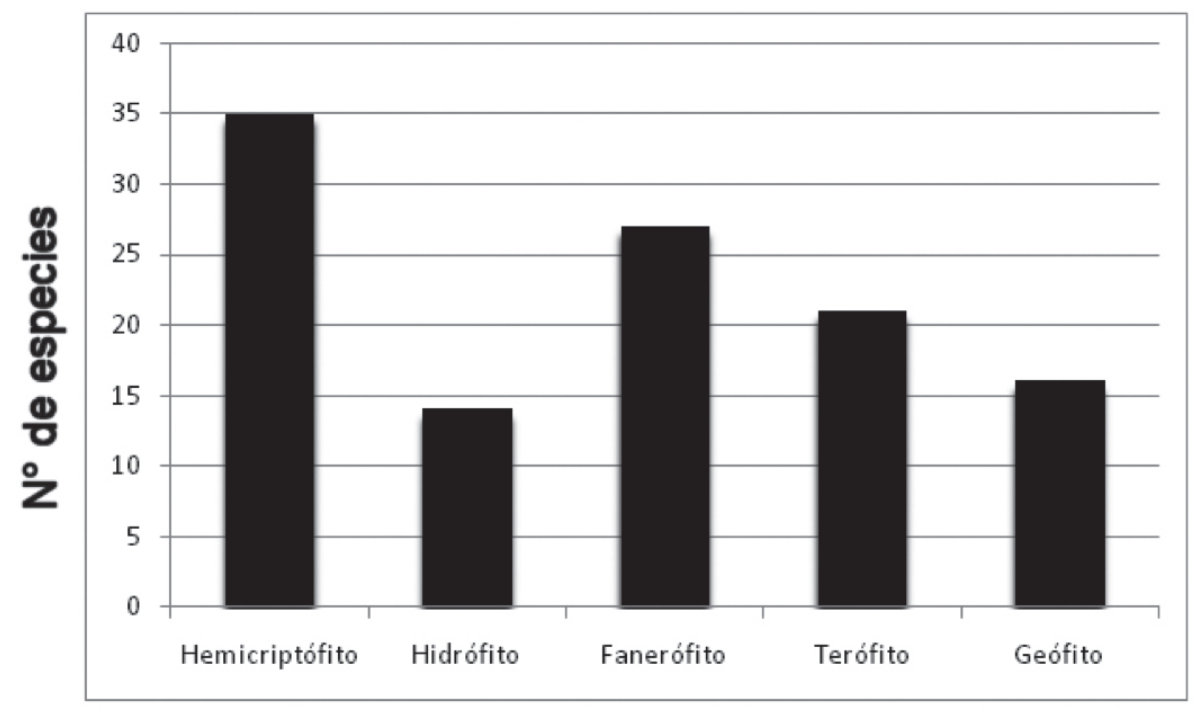

Forma de vida

Figura 2. Formas de vida de acuerdo a la clasificación de Raunkaier (1934) encontradas en la Laguna Avendaño. / Life forms according to Raunkaier (1934) found in Lagoon Avendaño.

TABLA 1. Resumen de la flora vascular del área en estudio. / Summary of the vascular flora of the area.

\begin{tabular}{lcccc}
\hline & $\begin{array}{c}\text { ESPECIES } \\
\text { ENDÉMICAS }\end{array}$ & EsPeCIES NATIVAS & $\begin{array}{c}\text { EsPeCIES } \\
\text { INTRODUCIDAS }\end{array}$ & Total ESPECIES \\
\hline Pteridophyta & 2 & 4 & - & 6 \\
Dicotyledoneae & 11 & 34 & 32 & 77 \\
Monocotyledoneae & 2 & 21 & 7 & 30 \\
\hline Total & 15 & 59 & 39 & 113 \\
\hline
\end{tabular}


Flora de la Laguna Avendaño, Quillón, Chile: BAEzA, C. ET AL.

TABLA 2. Familias con más de cinco especies. / Families with more than five species.

\begin{tabular}{lccc}
\hline FAMILIA & $\begin{array}{c}\text { EsPeCIES NATIVAS O } \\
\text { ENDÉMICAS }\end{array}$ & $\begin{array}{c}\text { EsPECIES } \\
\text { INTRODUCIDAS }\end{array}$ & TOTAL \\
\hline Asteraceae & 6 & 6 & 12 \\
Poaceae & 7 & 5 & 12 \\
Cyperaceae & 7 & 1 & 8 \\
Polygonaceae & 2 & 4 & 6 \\
Plantaginaceae & 3 & 2 & 5 \\
Fabaceae & 2 & 5 & 7 \\
\hline
\end{tabular}

TABLA 3. Plantas vasculares acuáticas presentes en la Laguna Avendaño. / Aquatic vascular plants in the Avendaño Lagoon.

\begin{tabular}{lccc}
\hline ESPECIE & CONDICIÓN & HÁBITO & ORIGEN \\
\hline Azolla filiculoides & libre flotante & hierba anual & nativa \\
Callitriche lechleri & arraigada sumergida & hierba anual & nativa \\
Ceratophyllum demersum & arraigada sumergida & hierba perenne & nativa \\
Egeria densa & arraigada sumergida & hierba perenne & introducida \\
Ludwigia peploides & arraigada emergente & hierba perenne & nativa \\
Myriophyllum aquaticum & arraigada sumergida & hierba perenne & nativa \\
Phragmites australis & arraigada emergente & hierba perenne & introducida \\
Potamogeton lucens & arraigada sumergida & hierba perenne & nativa \\
Potamogeton pusillus & arraigada sumergida & hierba anual o bienal & nativa \\
Schoenoplectus californicus & arraigada emergente & hierba perenne & nativa \\
Schoenoplectus pungens & arraigada emergente & hierba perenne & nativa \\
Typha angustifolia & arraigada emergente & hierba perenne & nativa \\
Utricularia gibba & arraigada sumergida & hierba perenne & nativa \\
Wolffia brasiliensis & libre flotante & hierba anual & nativa \\
\hline
\end{tabular}

\section{Plantas VAsculares ACUÁticas}

Entre las plantas vasculares acuáticas se pueden distinguir aquellas libres flotantes, las arraigadas al sustrato sumergidas y las arraigadas al sustrato emergentes (Tabla 3). Además, existe otra forma de crecimiento, las plantas palustres, cuyo hábitat es muy húmedo y pasan gran parte del año con sus raíces en el agua. Estas son abundantes en el lugar estudiado y corresponden a hierbas perennes o anuales, cubriendo a veces una importante área del espejo de agua.

También hay plantas trepadoras o volubles, como es el caso de Mikania mendocina, Calystegia sepium, Convolvulus hermanniae, Muehlenbeckia hastulata y Cissus striata.

\section{Plantas cultivadas}

Plantas cultivadas que intencionalmente han sido llevadas al lugar, ya sea como ornamentales o de uso agrícola, son: Asparagus officinalis (espárrago), Eucalyptus camaldulensis (eucalipto), Laurus nobilis (laurel de cocina),
Populus deltoides (álamo), Pseudotsuga menziesii (pino de Oregón), Salix matsudana (sauce torcido), Vitis vinifera (vid), Zantedeschia aethiopica (cala).

ESPECIES ENDÉMICAS

El 13,2 \% de las especies presentes en el área de estudio son endémicas y ninguna de ellas se encuentra listada en algún estado de conservación (IUCN 2015), ellas son: Adiantum scabrum, Azara integrifolia, Chusquea quila, Colliguaja dombeyana, Crinodendron patagua, Cryptocarya alba, Equisetum pyramidale, Escallonia pulverulenta, Lithrea caustica, Myrceugenia lanceolata, Myrceugenia obtusa, Otholobium glandulosum, Peumus boldus, Puya chilensis y Quillaja saponaria.

Comparación ENTRE LOS Sitios DE MUESTREO

El dendrograma obtenido entre sitios demostró que los sitios C y D fueron los más similares entre sí (Fig. 3), mientras que el sitio A fue el más diferente. El índice de 
correlación cofenética sugirió una alta correlación entre la matriz de similitud y el dendrograma obtenido $(\mathrm{r}=0.8814)$. El análisis SIMPROF reveló que los sitios C y D son significativamente similares en su composición, en relación al resto de los sitios analizados. Todos los sitios presentaron sobre un $30 \%$ de especies introducidas, excepto el sitio A.

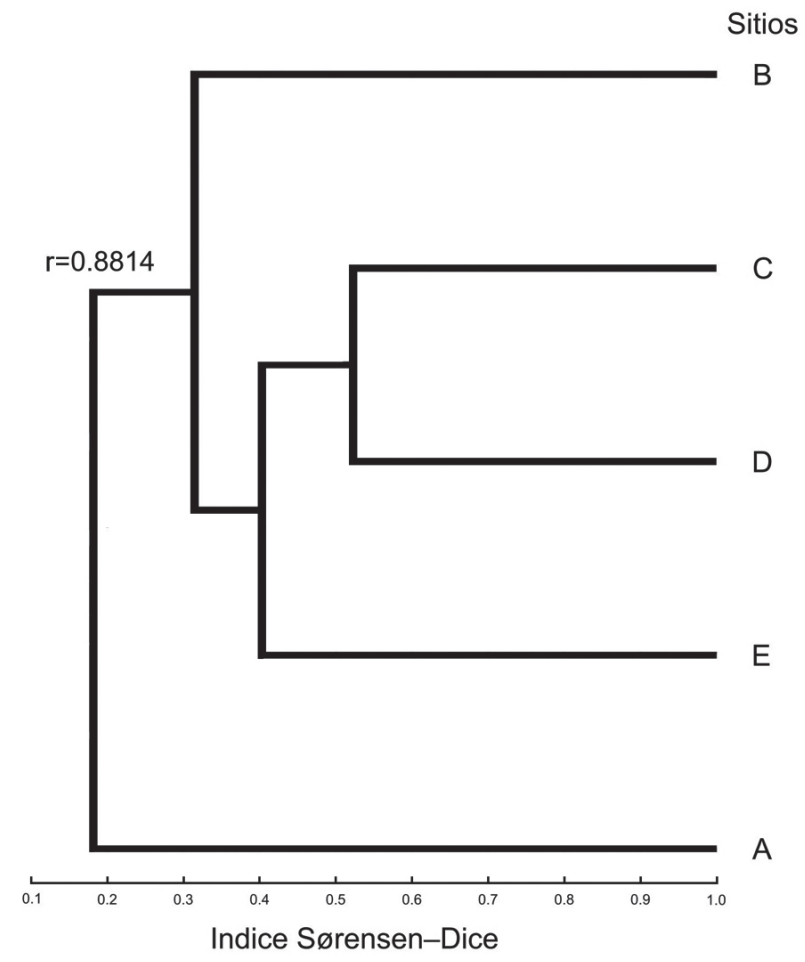

Figura 3. Dendrograma generado vía UPGMA e índice de Sorensen-Dice. / Dendrogram generated via UPGMA and Sorensen-Dice index.

\section{DISCUSIÓN}

El área de estudio presenta un número total de 15 especies endémicas $(13,3 \%), 59$ especies nativas $(52,2 \%)$ y 39 especies introducidas $(34,5 \%)$. Las familias mejor representadas son Poaceae, Asteraceae, Cyperaceae y Scrophulariaceae. En el cuerpo de agua las especies dominantes son Ceratophyllum demersum, Egeria densa y Potamogeton lucens. En los sectores aledaños a laguna Avendaño dominan Phragmites australis, Schoenoplectus californicus, Salix viminalis, Myrceugenia lanceolata y Typha angustifolia, entre otras. La mayoría de las especies endémicas corresponden a árboles o arbustos, probablemente esta condición de vida ha permitido su permanencia en los lugares donde están creciendo. De
Esta localidad reflejó el mayor nivel de antropización con valores cercanos a un 55\% de especies introducidas (Fig. 4). En cambio, los sitios con menor nivel de antropización, en términos de un mayor porcentaje de especies nativas (25.6\%) y endémicas (41.1\%), se presentó en el sitio B y sitio D, respectivamente (Fig. 4).

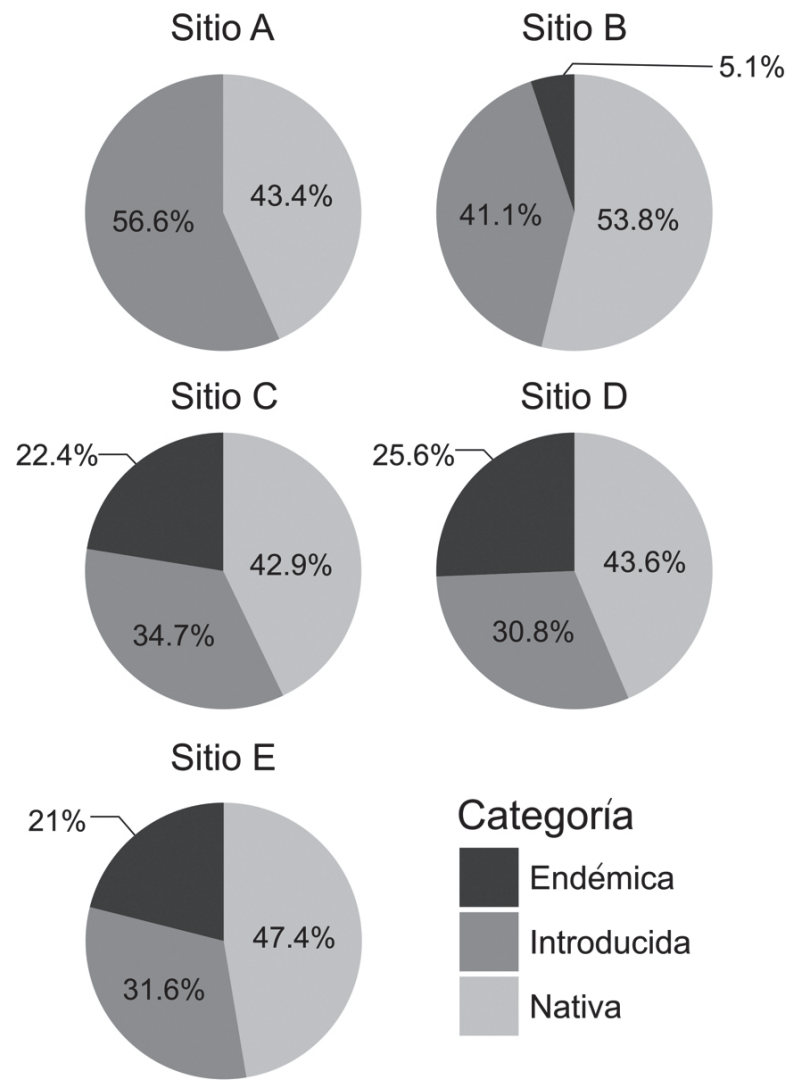

Figura 4. Riqueza taxonómica relativa en cada sitio de muestreo. / Relative taxonomic weights at each sampling site.

las 15 especies endémicas, solamente Adiantum scabrum y Puya chilensis presentan algún problema de conservación y ambas están en la categoría de preocupación menor.

Interesantemente, los patrones presentes en el dendrograma reflejarían que la similitud entre sitios estaría generada en función de sus niveles de antropización, los que a su vez estarían reflejados en sus proporciones individuales de flora nativa, endémica o introducida. Por una parte, los sitios del Dr. Ferrari (C), Antupahuil (D) y Brazo Bravo (E) presentaron los mayores niveles de similitud (Fig. 3), los cuales se explicarían por sus altos porcentajes de especies nativas y endémicas en relación al de especies introducidas (Fig. 4). Estos sitios son de propiedad particular, donde ha 
existido un limitado nivel de antropización dado a que su único acceso es de tipo restringido o solo accesible a través de embarcaciones. En estas áreas es posible aun distinguir vestigios de la vegetación autóctona, posiblemente aledaña a la laguna hace décadas antes de empezar el proceso de urbanización costera. Por el contrario, el área más disimilar correspondió al Balneario Municipal (A), la que presentó una alta proporción de especies introducidas y nativas (56,6\% y un 43,4\%; Fig. 4), y siendo el único sitio analizado sin especies endémicas. Tal sector se encuentra abierto al público durante gran parte del año, recibiendo un alto número de visitantes durante la temporada estival. Esto favorecería un flujo y establecimiento de especies introducidas con alto potencial invasivo. El nivel de antropización observado es tal que en este sitio se observó una gran biomasa de Myriophyllum aquaticum en el espejo de agua durante el censo de especies, lo que podría representar a futuro un gran problema de eutrofización para esta laguna (Rodríguez \& Dellarossa 1987).

Estos resultados respaldan la incidencia del efecto antrópico en la introducción y colonización de plantas introducidas, lo que puede producir el desplazamiento y posible extinción local de especies nativas y endémicas desde sus ambientes naturales. En este sentido, es recomendable mantener una buena gestión y organización en la habilitación de áreas recreativas en la laguna Avendaño, de modo de disminuir el impacto de pérdida de biodiversidad en estos ambientes naturales.

\section{CATÁlOGO DE LA FLORA VASCULAR DE LAGUNA AVENDAÑO}

\section{PTERIDOPHYTA}

AZOLLACEAE

Azolla filiculoides Lam.

Hierba anual, libre flotante en la superficie del agua. Nativa del continente americano.

\section{BLECHNACEAE}

Blechnum hastatum Kaulf.

Hierba perenne, crece en el piso del bosque y en lugares abiertos. Nativa de Chile y Argentina.

\section{EQUISETACEAE}

Equisetum bogotense Kunth

Hierba perenne, palustre, crece en lugares húmedos muy cerca del agua. Nativa del continente americano.

Equisetum pyramidale Goldm.

Hierba perenne, palustre, de lugares muy húmedos. Endémica de Chile.

\section{PTERIDACEAE}

Adiantum chilense Kaulf.

Hierba perenne, crece en el piso del bosque. Nativa de Chile y Argentina.

Adiantum scabrum Kaulf.

Hierba perenne, crece en el piso del bosque. Endémica de Chile.

\section{ANGIOSPERMAE - DYCOTYLEDONEAE}

ANACARDIACEAE

Lithrea caustica (Molina) Hook. \& Arn. Árbol endémico de Chile

\section{APIACEAE}

Daucus carota L. Hierba bienal, introducida, originaria de Europa.

Eryngium paniculatum Cav. \& Dombey ex F. Delaroche Hierba perenne, nativa de Sudamérica.

Hydrocotyle ranunculoides L.f.

Hierba perenne, palustre, nativa de Sudamérica.

Hydrocotyle umbellata $\mathrm{L}$.

Hierba perenne, palustre, nativa de Sudamérica.

\section{ASTERACEAE}

Arctotheca calendula (L.) Levyns

Hierba anual, introducida, originaria de África del Sur.

Baccharis linearis (Ruiz \& Pav.) Pers. Arbusto, nativo de Chile y Argentina.

Baccharis sagittalis (Less.) DC. Subarbusto, nativo de Chile y Argentina.

Bidens aurea (Aiton) Sherff

Hierba perenne, introducida, originaria de América del Norte.

Chamaemelum mixtum (L.) All.

Hierba anual, introducida, originaria de Europa.

Cichorium intybus L.

Hierba anual o bienal, introducida, originaria de Europa.

Conyza sumatrensis (Retz.) E. Walker var. leiotheca (S.F. Blake) Pruski \& G. Sancho

Hierba anual, nativa de Sudamérica.

Crepis capillaris (L.) Wallr.

Hierba anual, introducida, nativa de Europa.

Hypochaeris glabra $\mathrm{L}$.

Hierba anual, introducida, nativa de Europa.

Madia sativa Molina

Hierba anual, nativa de Argentina y Chile.

Mikania mendocina Phil.

Hierba perenne, voluble, nativa de Argentina y Chile.

Solidago chilensis Meyen

Hierba perenne, nativa de Sudamérica.

\section{CARYOPHYLLACEAE}

Spergula arvensis $\mathrm{L}$. 
Hierba anual, introducida, originaria de Europa.

\section{CELASTRACEAE}

Maytenus boaria Molina

Árbol nativo de Sudamérica.

\section{CERATOPHYLLACEAE}

Ceratophyllum demersum $\mathrm{L}$.

Hierba perenne, acuática, arraigada sumergida, nativa de Sudamérica.

\section{CONVOLVULACEAE}

Calystegia sepium (L.) R. Br. var. americana (Sims) Kitag. Hierba perenne, voluble, palustre, introducida, originaria de Norteamérica.

Convolvulus hermanniae L'Hér.

Hierba perenne, voluble, nativa de Sudamérica.

\section{ELAEOCARPACEAE}

Aristotelia chilensis (Molina) Stuntz

Árbol, nativo de Argentina y Chile.

Crinodendron patagua Molina

Árbol, endémico de Chile. Es común en el sector oeste, frente al Balneario Municipal.

\section{ESCALLONIACEAE}

Escallonia pulverulenta (Ruiz \& Pav.) Pers.

Árbol pequeño o arbusto, endémico de Chile.

\section{EUPHORBIACEAE}

Colliguaja dombeyana A.Juss.

Arbusto, endémico de Chile.

\section{FABACEAE}

Acacia caven (Molina) Molina

Árbol pequeño, nativo de Sudamérica.

Acacia dealbata Link

Árbol introducido, originario de Australia.

Galega officinalis L.

Hierba perenne, introducida, originaria de Europa.

Lotus corniculatus L.

Hierba perenne, introducida, originaria de Europa.

Otholobium glandulosum (L.) J.W. Grimes

Árbol pequeño o arbusto, endémico de Chile.

Teline monspessulana (L.) K. Koch

Arbusto introducido, originario de Europa.

Trifolium arvense $\mathrm{L}$.

Hierba anual, introducida, originaria de Europa.

\section{GUNNERACEAE}

Gunnera tinctoria (Molina) Mirb.

Hierba perenne, palustre, nativa de Argentina y Chile.
HALORAGACEAE

Myriophyllum aquaticum (Vell.) Verdc.

Hierba perenne, acuática, arraigada sumergida, nativa de Sudamérica.

\section{HYPERICACEAE}

Hypericum perforatum $\mathrm{L}$.

Hierba perenne, introducida, originaria de Europa.

\section{LAMIACEAE}

Mentha aquatica $\mathrm{L}$.

Hierba perenne, introducida, originaria de Europa.

Mentha pulegium L.

Hierba perenne, introducida, originaria de Europa.

Prunella vulgaris $\mathrm{L}$.

Hierba perenne, introducida, originaria de Europa.

LAURACEAE

Cryptocarya alba (Molina) Looser

Árbol, endémico de Chile.

\section{LENTIBULARIACEAE}

Utricularia gibba $\mathrm{L}$.

Hierba perenne, acuática, arraigada sumergida, es una pequeña planta carnívora. Nativa de Sudamérica.

\section{LINACEAE}

Linum bienne $\mathrm{L}$.

Hierba bienal, introducida, originaria de Europa.

\section{LYTHRACEAE}

Lythrum hyssopifolia $\mathrm{L}$.

Hierba anual, introducida, originaria de Europa.

MONIMIACEAE

Peumus boldus Molina

Árbol endémico de Chile.

\section{MYRTACEAE}

Luma apiculata (DC.) Burret

Árbol nativo de Argentina y Chile.

Myrceugenia exsucca (DC.) O. Berg

Árbol nativo de Argentina y Chile.

Myrceugenia lanceolata (Juss. ex J.St.-Hil.) Kausel

Arbusto endémico de Chile.

Myrceugenia obtusa (DC.) O. Berg

Árbol pequeño o arbusto, endémico de Chile.

\section{ONAGRACEAE}

Epilobium subdentatum (Meyen) Lievens \& Hoch

Hierba anual, nativa de Argentina y Chile.

Ludwigia peploides (Kunth) P.H. Raven subsp. montevidensis (Spreng.) P.H. Raven 
Hierba perenne, acuática, arraigada emergente, nativa de Sudamérica.

Oenothera stricta Ledeb. ex Link

Hierba anual o bienal, nativa de Argentina y Chile.

\section{OROBANCHACEAE}

Bellardia trixago (L.) All.

Hierba anual, introducida, originaria de Europa.

OXALIDACEAE

Oxalis perdicaria (Molina) Bertero

Hierba perenne, nativa de Sudamérica.

\section{PLANTAGINACEAE}

Callitriche lechleri (Hegelm.) Fassett

Hierba anual, acuática, arraigada sumergida, nativa de Sudamérica.

Limosella australis $\mathrm{R}$. Br.

Hierba anual, palustre, nativa de Argentina y Chile.

Plantago lanceolata $\mathrm{L}$.

Hierba perenne, introducida, originaria de Europa.

Stemodia durantifolia (L.) Sw.

Hierba perenne, palustre, nativa de Sudamérica.

Veronica anagallis-aquatica $\mathrm{L}$.

Hierba perenne, introducida, originaria de Europa y Asia.

\section{POLYGONACEAE}

Muehlenbeckia hastulata (Sm.) I.M. Johnst.

Arbusto trepador, nativo de Argentina y Chile.

Polygonum hydropiperoides Michx.

Hierba perenne, palustre, nativa de Sudamérica.

Polygonum lapathifolium $\mathrm{L}$.

Hierba perenne, introducida, originaria de Europa.

Polygonum persicaria L.

Hierba anual, introducida, originaria de Europa.

Rumex acetosella L.

Hierba perenne, introducida, originaria de Europa.

Rumex crispus L.

Hierba perenne, palustre, introducida, originaria de

Europa.

PRIMULACEAE

Anagallis alternifolia Cav.

Hierba perenne, palustre, nativa de Argentina y Chile.

QUILLAJACEAE

Quillaja saponaria Molina

Árbol endémico de Chile.

\section{RHAMNACEAE}

Colletia hystrix Clos

Arbusto, nativo de Argentina y Chile.
ROSACEAE

Rosa rubiginosa $\mathrm{L}$.

Arbusto introducido, originario de Europa.

Rubus ulmifolius Schott

Arbusto introducido, originario de Europa.

RUBIACEAE

Oldenlandia salzmannii (DC.) Benth. \& Hook. f. ex B.D. Jacks

Hierba anual, palustre, nativa de Sudamérica.

SALICACEAE

Azara integrifolia Ruiz et Pav.

Árbol pequeño o arbusto, endémico de Chile.

Salix viminalis L.

Arbusto introducido, palustre, originario de Europa y Asia.

\section{SCROPHULARIACEAE}

Verbascum thapsus L.

Hierba bienal, introducida, originaria de Europa y Asia.

Verbascum virgatum Stokes

Hierba bienal, introducida, originaria de Europa.

SOLANACEAE

Nierembergia repens Ruiz \& Pav.

Hierba perenne, nativa de Sudamérica.

VERBENACEAE

Verbena litoralis Kunth

Hierba perenne, nativa de Sudamérica.

VITACEAE

Cissus striata Ruiz \& Pav.

Arbusto trepador, nativo de Argentina y Chile.

\section{ANGIOSPERMAE - MONOCOTYLEDONEAE}

BROMELIACEAE

Puya chilensis Molina

Hierba perenne, endémica de Chile.

\section{CYPERACEAE}

Cyperus difformis $\mathrm{L}$.

Hierba perenne, palustre, introducida, originaria de Europa.

Cyperus eragrostis Lam.

Hierba perenne, palustre, nativa de Sudamérica.

Cyperus rigens J. Presl. \& C. Presl.

Hierba perenne, palustre, nativa de Sudamérica.

Eleocharis bonariensis Nees

Hierba perenne, palustre, nativa de Sudamérica.

Eleocharis melanostachys (D’Urv.) C.B. Clarke

Hierba perenne, palustre, nativa de Argentina y Chile. 
Schoenoplectus californicus (C.A. Mey.) Soják

Hierba perenne, acuática, arraigada emergente, nativa de Sudamérica. Es común en el sector oeste, frente al Balneario Municipal.

Schoenoplectus pungens (Vahl) Palla

Hierba perenne, acuática, arraigada emergente, nativa de Sudamérica.

Uncinia phleoides (Cav.) Pers.

Hierba perenne, nativa de Argentina y Chile.

\section{HYDROCHARITACEAE}

Egeria densa Planch.

Hierba perenne, acuática, arraigada sumergida, introducida, originaria de América del Sur.

\section{JUNCACEAE}

Juncus bufonius L.

Hierba perenne, palustre, nativa de Sudamérica.

Juncus imbricatus Laharpe

Hierba perenne, palustre, nativa de Sudamérica.

Juncus pallescens Lam.

Hierba perenne, palustre, nativa de Sudamérica.

Juncus procerus E. Mey.

Hierba perenne, palustre, nativa de Argentina y Chile.

\section{LEMNACEAE}

Wolffia brasiliensis Wedd.

Hierba perenne, acuática flotante, nativa de Sudamérica.

\section{POACEAE}

Bromus lithobius Trin.

Hierba perenne, nativa de Argentina y Chile.

Bromus secalinus L.

Hierba anual, introducida, originaria de Europa.

Cynodon dactylon (L.) Pers.

Hierba perenne, introducida, originaria de África tropical.

Chusquea quila Kunth

Arbusto, endémico de Chile.

Holcus lanatus L.

Hierba anual, introducida, originaria de Europa.

Imperata condensata Steud.

Hierba perenne, nativa de Sudamérica.

Paspalum dasypleurum Kunze ex E. Desv.

Hierba perenne, nativa de Sudamérica.

Phragmites australis (Cav.) Trin. ex Steud.

Hierba perenne, acuática, arraigada emergente, nativa de

Sudamérica.

Polypogon monspeliensis (L.) Desf.

Hierba anual, introducida, originaria de Europa.

Setaria parviflora (Poir.) M. Kerguélen

Hierba perenne, nativa de Sudamérica.

Sorghum halepense (L.) Pers.

Hierba perenne, introducida, originaria de África.
Sporobolus indicus (L.) R. Br.

Hierba perenne, nativa de Sudamérica.

\section{POTAMOGETONACEAE}

Potamogeton lucens $\mathrm{L}$.

Hierba perenne, acuática, arraigada sumergida, nativa de Sudamérica.

Potamogeton pusillus L.

Hierba anual o bienal, acuática, arraigada sumergida, nativa de Sudamérica.

\section{TYPHACEAE}

Typha angustifolia $\mathrm{L}$.

Hierba perenne, acuática, arraigada emergente, nativa de Sudamérica.

\section{AGRADECIMIENTOS}

DIUC No 201.111.028-1.0 Universidad de Concepción (dirigido por Víctor Dellarossa) y proyecto Flora de Chile. Oscar Toro-Núñez agradece al proyecto Fondecyt $\mathrm{N}^{\circ}$ 3160453. A C. Muñoz-Escobar por su ayuda con los análisis estadísticos. A Tobias Marczewski, Oscar Henríquez y Don Fernando, quien condujo la embarcación para hacer los muestreos.

\section{REFERENCIAS}

Baeza, C., Marticorena, C., Rodríguez, R. 1999. Catálogo de la flora vascular del Monumento Natural Contulmo, Chile. Gayana Botánica 56(2): 125-135.

Clarke, K., Gorley, R. 2006. PRIMER v6: User Manual/Tutorial. PRIMER-E, Plymouth. 192 pp.

Clarke, K., Sommerfield, P., Gorley, R. 2008. Testing of null hypotheses in exploratory community analyses similarity profiles and biota-environment linkage. Journal of Experimental Marine Biology and Ecology 366: 56-69.

Cronquist, A. 1981. An integrated system of classification of flowering plants. Columbia University Press, New York, USA. $1262 \mathrm{pp}$.

Dellarossa, V. 1987. Producción primaria en los lagos costeros de la Octava Región. Tesis de Magíster con mención en Botánica, Facultad de Ciencias Naturales y Oceanografía. Universidad de Concepción. Concepción, Chile. 104 pp.

Dellarossa, V. 1998. Producción primaria anual en sistemas de alta producción biológica. Tesis de Doctor en ciencias mención en Botánica, Universidad de Concepción. Concepción, Chile. 310 pp.

DicE, L. 1945. Measures of the amount of ecologic association between species. Ecology 26(3): 297-302.

Dray, S., Dufour, A. 2007. The ade4 package: implementing the duality diagram for ecologist. Journal of Statistical Software 22(4): 1-20.

Fuenzalida, H. 1965. Orografía. En: CORFO. Geografía económica de Chile. Texto refundido pp. 6-34. Corporación 
de Fomento de la Producción. Santiago, Chile.

GAJARDO, R. 1994. La vegetación natural de Chile. Clasificación y distribución geográfica. Ed. Universitaria. Santiago. 165 pp.

Hammer, O., Harper, D., Ryan, D. 2001. PAST: Paleontological statistics software package for education and data analysis. Paleontologia electronica 4(1): 1-9 pp.

IUCN. 2015. The IUCN Red List of Threatened Species. Version 2015-4. URL: http://www.iucnredlist.org. Acceded: November 19, 2015.

Luebert, F., Pliscoff, P. 2006. Sinopsis bioclimática y vegetacional de Chile. Ed. Universitaria, Santiago de Chile. 316 pp.

Oksanen, J., Blanchet, G., Friendly, M., Kindt, R., Legendre, P., McGlinn, D., Minchin, P., O’Hara, R., Simpson, G., Solymos, P., Stevens, H., Szoecs, E., Wagner, H. 2017. Vegan: Community ecology package. $\mathrm{R}$ package version 2.4-2. URL: https://CRAN.R-project.org/package=vegan

Ramírez, C., San Martín, J., San Martín, C., Contreras, D. 1987. Estudio florístico y vegetacional de la Laguna El Peral, Quinta Región de Chile. Revista de Geografía de Valparaíso 18: 105-120.

Raunkaier, C. 1934. The life forms of plants and statistical plant geography. Clarendon Press, Oxford. 632 pp.

R Core Team. 2017. R: A language and environment for statistical computing. $\mathrm{R}$ foundations for statistical computing, Vienna, Austria.

Rodríguez, R., Dellarossa, V. 1998. Plantas Vasculares acuáticas en la Region del Biobío. Universidad de Concepción, Concepción 38 pp.
Rodríguez, R., Grau, J., Baeza, C., Davies, A. 2008. Lista comentada de las plantas vasculares de Los Nevados de Chillán. Gayana Botánica 65(2): 153-197.

SAN Martín, J. 1989. Vegetación y flórula ribereña en el Río Purapel, VII Región. Maule UC 12: 54-63.

San Martín, C., Contreras, D., San Martín, J., Ramírez, C. 1992. Vegetación de las marismas del centro-sur de Chile. Revista Chilena de Historia Natural 65: 327-342.

San Martín, C., Ramírez, C., San Martín, J., Villaseñor, R. 2001. Flora y vegetación del Estero Reñaca (V Región, Chile). Gayana Botánica 58(1): 31-46.

Sokal, R., Rolph, R. 1962. The comparison of dendrograms by objective methods. Taxon 11: 33-40.

Sørensen, T. 1948. A method of establishing groups of equal amplitude in plant sociology based on similarity of species and its application to analyses of the vegetation on Danish commons. Kongelige Danske Videnskabernes Selskab 5(4): $1-34$

Whitaker, D., Christman, M. 2014. Clustsig: Significant cluster analysis. $\mathrm{R}$ package version 1.1. R package version 2.4-2. URL: https://CRAN.R-project.org/package=clustsig

Zúñiga-Reinoso, A., Muñoz-Escobar, C., Herández, C. 2013. Patrones y causas de estructuración geográfica latitudinal en los oribátidos (Acari: Oribatida) en Patagonia y Antártica. Revista Chilena de Historia Natural 86: 279289.

Recibido: 20.03.2018

Aceptado: 18.02.2019 